\title{
Rivaroxaban versus warfarin for treatment and prevention of recurrence of venous thromboembolism in African American patients: a retrospective cohort analysis
}

Olivia S. Costa ${ }^{1,2}$, Stanley Thompson ${ }^{3}$, Veronica Ashton ${ }^{4}$, Michael Palladino ${ }^{5}$, Thomas J. Bunz ${ }^{6}$ and Craig I. Coleman ${ }^{1,2^{*}}$

\begin{abstract}
Background: African Americans are under-represented in trials evaluating oral anticoagulants for the treatment of acute venous thromboembolism (VTE). The aim of this study was to evaluate the effectiveness and safety of rivaroxaban versus warfarin for the treatment of VTE in African Americans.

Methods: We utilized Optum ${ }^{\oplus}$ De-Identified Electronic Health Record data from 11/1/2012-9/30/2018. We included African Americans experiencing an acute VTE during a hospital or emergency department visit, who received rivaroxaban or warfarin as their first oral anticoagulant within 7-days of the acute VTE event and had $\geq 1$ provider visit in the prior 12-months.

Differences in baseline characteristics between cohorts were adjusted using inverse probability-of-treatment weighting based on propensity scores (standard differences $<0.10$ were achieved for all covariates). Our primary endpoint was the composite of recurrent VTE or major bleeding at 6-months. Three- and 12-month timepoints were also assessed. Secondary endpoints included recurrent VTE and major bleeding as individual endpoints. Cohort risk was compared using Cox regression and reported as hazard ratios (HRs) with 95\% confidence intervals (Cls).

Results: We identified 2097 rivaroxaban and 2842 warfarin users with incident VTE. At 6-months, no significant differences in the composite endpoint ( $\mathrm{HR}=0.96,95 \% \mathrm{Cl}=0.75-1.24)$, recurrent $\mathrm{VTE}(\mathrm{HR}=1.02,95 \% \mathrm{Cl}=0.76-1.36)$ or major bleeding alone $(\mathrm{HR}=0.93,95 \% \mathrm{Cl}=0.59-1.47)$ were observed between cohorts. Analysis at 3- and 12-months provided consistent findings for these endpoints.
\end{abstract}

Conclusions: In African Americans experiencing an acute VTE, no significant difference in the incidence of recurrent VTE or major bleeding was observed between patients receiving rivaroxaban or warfarin.

Keywords: African Americans, Anticoagulants, Rivaroxaban, Venous thromboembolism, Warfarin

\footnotetext{
* Correspondence: craig.coleman@hhchealth.org

'Department of Pharmacy Practice, University of Connecticut School of

Pharmacy, 69 North Eagleville Road, Unit 3092, Storrs, CT 06269, USA

${ }^{2}$ Evidence-Based Practice Center, Hartford Hospital, Hartford, CT, USA

Full list of author information is available at the end of the article
}

(c) The Author(s). 2020 Open Access This article is licensed under a Creative Commons Attribution 4.0 International License, which permits use, sharing, adaptation, distribution and reproduction in any medium or format, as long as you give appropriate credit to the original author(s) and the source, provide a link to the Creative Commons licence, and indicate if changes were made. The images or other third party material in this article are included in the article's Creative Commons licence, unless indicated otherwise in a credit line to the material. If material is not included in the article's Creative Commons licence and your intended use is not permitted by statutory regulation or exceeds the permitted use, you will need to obtain permission directly from the copyright holder. To view a copy of this licence, visit http://creativecommons.org/licenses/by/4.0/ The Creative Commons Public Domain Dedication waiver (http://creativecommons.org/publicdomain/zero/1.0/) applies to the data made available in this article, unless otherwise stated in a credit line to the data. 


\section{Background}

There is ample evidence suggesting African Americans have an increased incidence of venous thromboembolism (VTE) and poorer disease outcomes compared to other racial groups [1-5]. Despite race-based differences in VTE incidence and prognosis, African Americans have been under-represented in randomized controlled trials (RCTs) evaluating non-vitamin $\mathrm{K}$ oral anticoagulants (NOACs) for the management of VTE [6-10]. As a result, there is a scarcity of data evaluating NOACs for the treatment and secondary prevention of VTE.

The objective of this study was to evaluate the effectiveness and safety of rivaroxaban versus warfarin in the treatment and prevention of recurrent VTE in African Americans managed in routine practice.

\section{Methods}

We performed a retrospective cohort analysis using United States (US) Optum ${ }^{\circ}$ De-Identified Electronic Health Record (EHR) data from November 1, 2012 to September 30, 2018 [11]. The Optum EHR database includes longitudinal patient-level medical record data for 97 million patients seen at 700 hospitals and 7000 clinics across the United States. The database includes records of prescriptions as prescribed and administered, lab results, vital signs, body measurements, diagnoses, procedures, and information derived from clinical notes using natural language processing. This database contains data on insured and uninsured patients of all ages and races to provide a representative sample of all African American patients with acute VTE in the US.

To be included in this study patients had to be African American, admitted to the hospital, emergency department or observation unit for acute deep vein thrombosis (DVT) or pulmonary embolism (PE), have received rivaroxaban or warfarin as their first oral anticoagulant (OAC) within 7-days of the acute VTE event (index date) and had at least one provider visit in the 12months prior to the acute VTE event (baseline period). We excluded patients with another indication for OAC use (i.e. atrial fibrillation, prophylaxis after hip/knee replacement, valvular heart disease).

Our primary endpoint for this analysis was the composite of recurrent VTE or major bleeding at 3-, 6- and 12 -months $[12,13]$. Recurrent VTE was defined as a subsequent hospitalization with a primary International Classification of Diseases-10th Revision (or cross-walked ICD-9 to ICD-10) diagnosis code for DVT (ICD-10 = $180-182)$ or PE (ICD-10 = 126) [12]. Major bleeding was defined as a subsequent hospitalization for a bleeding event using the validated Cunningham algorithm [13]. This algorithm defines a hospitalization as bleedingrelated based on the presence of an ICD-10 bleeding code in the primary position or the presence of select non-primary diagnosis ICD-10 codes accompanied by a billing code indicating a blood transfusion or processing of blood products for transfusion. Secondary endpoints included recurrent VTE and major bleeding as separate endpoints, as well as, intracranial hemorrhage (ICH), gastrointestinal bleeding (GIB) and genitourinary bleeding (GUB). Patients were followed for up to 12-months or until endpoint occurrence, end-of-EHR activity or through September 30, 2018 (an intent-to-treat approach).

Differences in baseline characteristics between the rivaroxaban and warfarin cohorts were adjusted for using inverse probability-of-treatment weighting (IPTW) based on propensity scores [14]. For the IPTW analysis, propensity scores (and subsequent patient weights) were estimated using generalized boosted models on the basis of 10,000 regression trees using the 'TWANG' package (version 1.5) and $\mathbf{R}$ statistical software version 3.4.3 (The R Project for Statistical Computing) which implements an automated, nonparametric machine learning method. The weights were derived to obtain estimates of the population average treatment effect. Variables entered in the generalized boosted modeling procedure (including demographics, comorbidities and concurrent non-anticoagulant medications) are depicted in Table 1 and were identified during the 12-month baseline period. The presence of residual differences in measured covariates following cohort weighting was assessed by calculating absolute standardized differences $(<0.1$ was considered well-balanced for each variable) [14].

Baseline categorical data were reported as percentages and continuous data as medians with $25,75 \%$ ranges. Endpoint incidence were reported as proportions (the number experiencing an event divided by total number of patients in the cohort). Weighted Cox proportional hazards regression analysis were performed using the 'SURVEY' package version 3.36 in R. Patients assigned weights at the extremes $(<1$ st or $>99$ th percentile) were recalibrated to the corresponding threshold values prior to weighted regression analyses [14]. The proportional hazard assumption was tested based on Schoenfeld residuals and was found valid for all endpoints.

We performed sensitivity analyses in which an ontreatment approach (i.e., patients followed for up to 12months or until endpoint occurrence, index OAC discontinuation [30-day permissible gap] or switch, end-of-EHR activity or through September 30,2018) was utilized and differences in baseline characteristics between the rivaroxaban and warfarin cohorts were adjusted using IPTW based on propensity scores. An additional sensitivity analysis was performed in which differences in baseline characteristics between the rivaroxaban and warfarin cohorts were adjusted by propensity score matching calculated via multivariable logistic regression using 'MatchIT' in $\mathbf{R}$. 
Table 1 Characteristics of the Rivaroxaban and Warfarin IntentTo-Treat Cohorts After IPTW

\begin{tabular}{llll}
\hline Rivaroxaban & Warfarin & Absolute \\
$N=2097$ & $N=2842$ & Standardized \\
$\%$ & $\%$ & Difference \\
\hline
\end{tabular}

\section{Demographics}

Age, median $(25,75 \%$ range)

$\begin{array}{lll}50(39,62) & \begin{array}{l}51(40, \\ 64)\end{array} & - \\ 45.5 & 43.6 & 0.04 \\ 29.6 & 29.2 & 0.01 \\ 13.1 & 13.7 & 0.02 \\ 4.3 & 4.7 & 0.02 \\ 7.5 & 8.9 & 0.05 \\ 56.4 & 56.4 & 0.00 \\ 18.6 & 19.1 & 0.01\end{array}$
vein thrombosis)

\section{Comorbidities}

$\begin{array}{llll}\text { Chronic obstructive } & 8.6 & 9.6 & 0.04 \\ \text { pulmonary disease } & & & \\ \text { Asthma } & 13.1 & 13.6 & 0.02 \\ \text { Heart Failure } & 5.1 & 5.4 & 0.01 \\ \text { Hypertension } & 53.5 & 55.2 & 0.03 \\ \text { Ischemic stroke } & 3.6 & 4.3 & 0.04 \\ \text { Diabetes } & 22.3 & 23.0 & 0.02 \\ \text { Dementia } & 2.6 & 3.3 & 0.04 \\ \text { Coronary artery disease } & 0.8 & 0.7 & 0.01 \\ \text { Carotid stenosis } & 0.6 & 0.8 & 0.03 \\ \text { Peripheral vascular disease } & 5.5 & 5.8 & 0.01 \\ \text { Myocardial infarction } & 5.2 & 5.9 & 0.03 \\ \text { Percutaneous coronary } & 3.5 & 4.1 & 0.03 \\ \text { intervention } & & & \\ \text { Coronary artery bypass } & 2.4 & 2.8 & 0.02 \\ \text { grafting } & & & \\ \text { Gastrointestinal bleed } & 0.3 & 0.5 & 0.02 \\ \text { Intracranial hemorrhage } & 0.0 & 0.3 & 0.07 \\ \text { Acute kidney injury } & 11.0 & 12.5 & 0.05 \\ \text { Other kidney injury } & 0.3 & 0.5 & 0.04 \\ \text { Inflammatory bowel disease } & 1.2 & 1.6 & 0.04 \\ \text { eGFR > 90 mL/minute } & 53.6 & 51.7 & 0.04 \\ \text { eGFR 60-89 mL/minute } & 30.9 & 30.8 & 0.00 \\ \text { eGFR 30-59 mL/minute } & 11.1 & 11.3 & 0.01 \\ \text { eGFR 15-29 mL/minute } & 1.9 & 2.7 & 0.06 \\ \text { eGFR < 15 mL/minute } & 1.7 & 2.6 & 0.06 \\ \text { eGFR unknown } & 0.7 & 0.7 & 0.01 \\ \text { Liver disease } & 1.6 & 1.6 & 0.00 \\ \text { Coagulopathy } & 3.1 & 3.5 & 0.02 \\ \text { Gastroesophageal reflux } & 18.9 & 18.8 & 0.00 \\ \text { disease } & & & \\ \text { Anemia } & 25.5 & 27.7 & 0.05\end{array}$

Table 1 Characteristics of the Rivaroxaban and Warfarin IntentTo-Treat Cohorts After IPTW (Continued)

\begin{tabular}{|c|c|c|c|}
\hline & $\begin{array}{l}\text { Rivaroxaban } \\
N=2097 \\
\%\end{array}$ & $\begin{array}{l}\text { Warfarin } \\
N=2842 \\
\%\end{array}$ & $\begin{array}{l}\text { Absolute } \\
\text { Standardized } \\
\text { Difference }\end{array}$ \\
\hline Sleep apnea & 10.4 & 10.2 & 0.01 \\
\hline Smoking & 27.4 & 27.4 & 0.00 \\
\hline Hemorrhoids & 2.3 & 2.6 & 0.02 \\
\hline Alcohol abuse & 0.4 & 0.3 & 0.02 \\
\hline Anxiety & 12.6 & 12.2 & 0.01 \\
\hline Depression & 1.6 & 1.8 & 0.02 \\
\hline Psychosis & 1.5 & 1.5 & 0.00 \\
\hline $\mathrm{BMI}<18.5 \mathrm{~kg} / \mathrm{m}^{2}$ & 1.9 & 1.9 & 0.00 \\
\hline BMI $18.5-24.9 \mathrm{~kg} / \mathrm{m}^{2}$ & 15.8 & 16.5 & 0.02 \\
\hline BMI $25.0-29.9 \mathrm{~kg} / \mathrm{m}^{2}$ & 24.8 & 24.6 & 0.01 \\
\hline BMI $30.0-34.9 \mathrm{~kg} / \mathrm{m}^{2}$ & 23.0 & 23.0 & 0.00 \\
\hline BMI $35.0-39.9 \mathrm{~kg} / \mathrm{m}^{2}$ & 14.7 & 14.3 & 0.01 \\
\hline $\mathrm{BMI} \geq 40 \mathrm{~kg} / \mathrm{m}^{2}$ & 18.4 & 18.4 & 0.00 \\
\hline $\mathrm{BMI}<18.5 \mathrm{~kg} / \mathrm{m}^{2}$ & 1.3 & 1.2 & 0.01 \\
\hline Rheumatoid arthritis & 6.2 & 5.8 & 0.02 \\
\hline Osteoarthritis & 18.9 & 19.9 & 0.03 \\
\hline Headache & 10.7 & 11.1 & 0.01 \\
\hline Diverticulitis & 3.8 & 3.8 & 0.00 \\
\hline H. pylori treatment & 0.4 & 0.6 & 0.02 \\
\hline Hypothyroidism & 0.8 & 0.9 & 0.00 \\
\hline Solid tumor & 9.5 & 10.2 & 0.02 \\
\hline Metastatic cancer & 3.5 & 3.8 & 0.01 \\
\hline Major surgery & 9.8 & 9.7 & 0.01 \\
\hline Varicose veins & 1.4 & 1.3 & 0.01 \\
\hline \multicolumn{4}{|l|}{ Comedications } \\
\hline Aspirin & 22.7 & 24.0 & 0.03 \\
\hline P2Y12 platelet inhibitor & 3.1 & 4.0 & 0.05 \\
\hline $\begin{array}{l}\text { Nonsteroidal anti- } \\
\text { inflammatory drug }\end{array}$ & 31.8 & 30.8 & 0.02 \\
\hline Celecoxib & 1.1 & 1.0 & 0.01 \\
\hline $\begin{array}{l}\text { Angiotensin-converting } \\
\text { enzyme inhibitor or receptor } \\
\text { blocker }\end{array}$ & 31.1 & 32.2 & 0.02 \\
\hline Beta-blocker & 23.5 & 25.3 & 0.04 \\
\hline Diltiazem & 1.8 & 2.0 & 0.01 \\
\hline Verapamil & 0.9 & 1.1 & 0.02 \\
\hline $\begin{array}{l}\text { Dihydropyridine calcium } \\
\text { channel blocker }\end{array}$ & 21.2 & 21.8 & 0.01 \\
\hline Loop diuretic & 11.0 & 11.9 & 0.03 \\
\hline Thiazide & 21.2 & 21.4 & 0.00 \\
\hline Digoxin & 0.5 & 0.8 & 0.04 \\
\hline Statin & 23.7 & 24.5 & 0.02 \\
\hline Other cholesterol medication & 2.0 & 2.1 & 0.01 \\
\hline Metformin & 11.7 & 11.5 & 0.01 \\
\hline
\end{tabular}


Table 1 Characteristics of the Rivaroxaban and Warfarin IntentTo-Treat Cohorts After IPTW (Continued)

\begin{tabular}{|c|c|c|c|}
\hline & $\begin{array}{l}\text { Rivaroxaban } \\
N=2097 \\
\%\end{array}$ & $\begin{array}{l}\text { Warfarin } \\
N=2842 \\
\%\end{array}$ & $\begin{array}{l}\text { Absolute } \\
\text { Standardized } \\
\text { Difference }\end{array}$ \\
\hline Sulfonylurea or glinides & 5.1 & 6.0 & 0.04 \\
\hline Thiazolidinediones & 0.5 & 0.6 & 0.02 \\
\hline $\begin{array}{l}\text { Dipeptidyl peptidase } 4 \\
\text { inhibitors }\end{array}$ & 1.5 & 1.4 & 0.01 \\
\hline $\begin{array}{l}\text { Glucagon-like peptide-1 } \\
\text { agonist }\end{array}$ & 0.5 & 0.3 & 0.03 \\
\hline Insulin & 7.8 & 8.9 & 0.04 \\
\hline $\begin{array}{l}\text { Selective serotonin reuptake } \\
\text { or serotonin-norepinephrine } \\
\text { reuptake inhibitor }\end{array}$ & 10.6 & 11.3 & 0.02 \\
\hline Other antidepressants & 9.0 & 9.7 & 0.03 \\
\hline Proton pump inhibitors & 21.4 & 22.6 & 0.03 \\
\hline Histamin-2 receptor antagonist & 8.4 & 9.0 & 0.02 \\
\hline Systemic corticosteroids & 17.8 & 18.8 & 0.03 \\
\hline Alpha-glucosidase inhibitor & 0.1 & 0.0 & 0.04 \\
\hline Hypnotic medication & 3.7 & 3.6 & 0.00 \\
\hline $\begin{array}{l}\text { Sodium-glucose cotransporter- } \\
2 \text { inhibitor }\end{array}$ & 0.3 & 0.2 & 0.02 \\
\hline
\end{tabular}

Each eligible rivaroxaban user was 1:1 propensity score matched using greedy nearest neighbor matching without replacement and a caliper $=0.25$ standard deviations of the propensity score. For sensitivity analysis, Cox proportional hazards regression analysis was performed using IBM SPSS version 25.0 (IBM Corp, Armonk, NY).

The report for this analysis was written to comply with the Reporting of Studies Conducted using Observational Routinely-Collected Health Data (RECORD) statement [15].

\section{Results}

In total, we identified 48,429 patients with a primary diagnosis for VTE with $\geq 1$ provider visit in the 12months prior to the index VTE event. Of these, 6158 were African Americans and 4939 were initiated on OAC with either rivaroxaban or warfarin within 7-days of the index event. The median age of included patients was 51 years, $56.4 \%$ o were female, $18.4 \%$ were morbidly obese (body mass index $\geq 40 \mathrm{~kg} / \mathrm{m}^{2}$ ), $9.7 \%$ had a history of prior major surgery, 3.7 and $9.9 \%$ and had a history of/active metastatic cancer or a solid tumor, respectively, and nearly 1 in 5 patients had a PE \pm DVT.

Following IPTW, patients were deemed well-balanced on all independent variables entered into the propensity-score model as demonstrated by absolute standardized differences between the rivaroxaban and warfarin users $<0.1$. Upon Cox regression, rivaroxaban use $(N=2097)$ was not associated with a statistically significant difference in the incidence of the composite endpoint of recurrent VTE or major bleeding at 3 -months $(4.57 \%$ versus $4.58 \%, \mathrm{HR}=1.08,95 \% \mathrm{CI}=0.82-$ $1.42)$, 6-months $(5.01 \%$ versus $5.84 \%, \mathrm{HR}=0.96,95 \% \mathrm{CI}=$ $0.75-1.24)$ or 12 -months $(5.82 \%$ versus $7.32 \%, \mathrm{HR}=0.93$, $95 \% \mathrm{CI}=0.74-1.16$ ) of follow-up (Table 2) compared to warfarin $(N=2842)$. No significant differences were observed between the cohorts for either of the components when evaluated separately at these same time points, nor were there significant differences in the incidence of ICH $(\mathrm{HR}=$ $0.66,95 \% \mathrm{CI}=0.12-3.59$ at 3 -months; $\mathrm{HR}=0.50,95 \% \mathrm{CI}=$ $0.10-2.50$ at 6 -months and $\mathrm{HR}=0.76,95 \% \mathrm{CI}=0.27-2.19$ at 12-months), GI ( $\mathrm{HR}=1.16,95 \% \mathrm{CI}=0.61-2.21$ at 3 -months; $\mathrm{HR}=0.84,95 \% \mathrm{CI}=0.47-1.51$ at 6 -months and $\mathrm{HR}=0.80$, $95 \% \mathrm{CI}=0.47-1.37$ at 12 -months) and GU bleeding (HR = $1.08,95 \% \mathrm{CI}=0.30-3.93$ at 3 -months; $\mathrm{HR}=0.80,95 \% \mathrm{CI}=$ $0.24-2.63$ at 6 -months and $\mathrm{HR}=0.88,95 \% \mathrm{CI}=0.29-2.63$ at 12-months). Sensitivity analyses 1) employing propensity score matching ( $N=2068$ users of rivaroxaban or warfarin per group) and 2) based upon an on-treatment analysis approach both provided similar results to the base-case analysis for the composite endpoint, recurrent VTE and major bleeding alone at each timepoint (Tables 3 and 4).

\section{Discussion}

This EHR-based study evaluated African American patients experiencing a VTE treated with rivaroxaban or warfarin in routine practice. Our analysis suggested there was no significant difference in the incidence of the composite endpoint of recurrent VTE or major bleeding between the treatment groups after 3-, 6- or 12-months of follow up. No significant differences were observed between the cohorts for either of the components when evaluated separately at these same time points, nor were there significant differences in the incidence of ICH, GI or GU bleeding. Our conclusions were also similar when an on-treatment approach and propensity score matching were utilized.

African American patients have been under-enrolled in RCTs evaluating NOACs for the treatment of VTE [7-10]. Moreover, no sub-analyses of an RCT has reported on the efficacy and/or safety of NOACs in a cohort of African American patients. Consequently, our present analysis provides important new data to aid in clinical decision-making. The findings of our study were generally consistent with those of the pooled EINSTEIN trial analysis which included a small portion $(2.6 \%)$ of black patients and the prospective, nonrandomized XALIA registry study $[5,9,16]$. In the pooled EINSTEIN trial analysis, rivaroxaban $(n=4151)$ was found to be non-inferior to enoxaparin/vitamin $\mathrm{K}$ antagonist (VKA) ( $n=4131)$ for the endpoint of recurrent VTE with a 2.1 and $2.3 \%$ incidence, respectively $(\mathrm{HR}=0.89 ; 95 \% \mathrm{CI}=$ 0.66-1.19). These results were echoed in XALIA which found no significant difference in recurrent VTE risk 
Table 2 Event Incidence and Hazard Ratios with 95\% Confidence Intervals for IPTW Analysis of the Rivaroxaban and Warfarin Cohorts Using an Intent-To-Treat Approach

\begin{tabular}{|c|c|c|c|}
\hline & $\begin{array}{l}\text { Rivaroxaban } \\
N=2097 \\
\mathrm{n}(\%)\end{array}$ & $\begin{array}{l}\text { Warfarin } \\
N=2842 \\
\mathrm{n}(\%)\end{array}$ & $\mathrm{HR}(95 \% \mathrm{Cl})$ \\
\hline \multicolumn{4}{|l|}{ 3-Month } \\
\hline Composite of recurrent venous thromboembolism or major bleeding & $96(4.58)$ & $130(4.57)$ & $1.08(0.82-1.42)$ \\
\hline Recurrent venous thromboembolism & $74(3.53)$ & $96(3.38)$ & $1.07(0.78-1.46)$ \\
\hline Major bleeding & $27(1.29)$ & $40(1.41)$ & $1.19(0.72-1.97)$ \\
\hline Intracranial hemorrhage & $2(0.10)$ & $5(0.18)$ & $0.66(0.12-3.59)$ \\
\hline Gastrointestinal bleeding & $17(0.81)$ & $24(0.84)$ & $1.16(0.61-2.21)$ \\
\hline Genitourinary bleeding & $4(0.19)$ & $6(0.21)$ & $1.08(0.30-3.93)$ \\
\hline \multicolumn{4}{|l|}{ 6-Month } \\
\hline Composite of recurrent venous thromboembolism or major bleeding & $105(5.01)$ & $166(5.84)$ & $0.96(0.75-1.24)$ \\
\hline Recurrent venous thromboembolism & $81(3.86)$ & $115(4.05)$ & $1.01(0.76-1.36)$ \\
\hline Major bleeding & $30(1.43)$ & $59(2.08)$ & $0.93(0.59-1.47)$ \\
\hline Intracranial hemorrhage & $2(0.10)$ & $7(0.25)$ & $0.50(0.10-2.50)$ \\
\hline Gastrointestinal bleeding & $18(0.86)$ & $37(1.30)$ & $0.84(0.47-1.51)$ \\
\hline Genitourinary bleeding & $4(0.19)$ & $9(0.32)$ & $0.80(0.24-2.63)$ \\
\hline \multicolumn{4}{|l|}{ 12-Month } \\
\hline Composite of recurrent venous thromboembolism or major bleeding & $122(5.82)$ & $208(7.32)$ & $0.93(0.74-1.16)$ \\
\hline Recurrent venous thromboembolism & $89(4.24)$ & $140(4.93)$ & $0.95(0.72-1.2)$ \\
\hline Major bleeding & $39(1.86)$ & $80(2.81)$ & $0.92(0.62-1.36)$ \\
\hline Intracranial hemorrhage & $5(0.24)$ & $13(0.46)$ & $0.76(0.27-2.19)$ \\
\hline Gastrointestinal bleeding & $21(1.00)$ & $47(1.65)$ & $0.80(0.47-1.37)$ \\
\hline Genitourinary bleeding & $5(0.24)$ & $10(0.35)$ & $0.88(0.29-2.63)$ \\
\hline
\end{tabular}

between rivaroxaban $(n=2619)(1.4 \%)$ and standard-ofcare management (typically parenteral bridging to a VKA) $(n=2149)(2.3 \%)$ of acute DVT $( \pm \mathrm{PE})$ in routine practice (propensity score-adjusted $\mathrm{HR}=0.91 ; 95 \% \mathrm{CI}=$ 0.54-1.54). While reductions in the risk of major bleeding was observed with rivaroxaban (1.0\%) compared to enoxaparin/VKA (1.7\%) in the EINSTEIN clinical trial program $(\mathrm{HR}=0.54 ; 95 \% \mathrm{CI}=0.37-0.79)$, no significant difference was observed in XALIA for rivaroxaban $(0.8 \%)$ versus standard-of-care $(2.1 \%)$ management (propensity score-adjusted $\mathrm{HR}=0.77$; $95 \% \mathrm{CI}=0.40-1.50)$.

This study has limitations worthy of discussion. First, multiple biases including misclassification, sampling and confounding bias are always important limitations in non-randomized, retrospective studies that may impact their internal validity [17]. We attempted to reduce the probability of misclassification bias by using validated coding schema to identify comorbidities and endpoints (when possible). Our methodology appeared to be effective given we reported recurrent VTE rates just slightly above previously published studies $[9,16$,
18] (which was anticipated as black patients have been hypothesized to be at a higher risk of thrombosis compared to other races [1-5]) as well as a similar incidence of major bleeding (range in prior studies: $0.77-$ 2.1\%) $[9,16,18]$. Second, we used EHR data for US patients [11]; and therefore, our results are most generalizable to a US population. Third, we did not calculate time in therapeutic international normalized ratio (INR) range for warfarin patients. While INR control is somewhat predictive of the effectiveness and safety of vitamin $\mathrm{K}$ antagonists, it is well known that VTE patients treated with warfarin in routine US clinical practice spend only $\sim 56 \%$ of their time during the first 3 months of treatment (when recurrent VTE is most likely) in the therapeutic INR range $[19,20]$ and African American patients may have among the poorest INR control [21]. Given the Optum EHR data used for this study covers patients throughout the US regardless of insurer (or no insurance), it is likely patients included in this study experienced at least similar suboptimal INR control. Fourth, results of the ontreatment analysis which was performed to supplement 
Table 3 Characteristics of the 1:1 Propensity Score Matched (Sensitivity Analysis) Rivaroxaban and Warfarin Cohorts

\begin{tabular}{|c|c|c|c|}
\hline & $\begin{array}{l}\text { Rivaroxaban } \\
N=2068 \\
\%\end{array}$ & $\begin{array}{l}\text { Warfarin } \\
N=2068 \\
\%\end{array}$ & $\begin{array}{l}\text { Absolute Standardized } \\
\text { Difference }\end{array}$ \\
\hline \multicolumn{4}{|l|}{ Demographics } \\
\hline Age, median ( $25,75 \%$ range) & $50(39,62)$ & $51(40,64)$ & - \\
\hline Age 18-49years & 46.32 & 48.21 & 0.04 \\
\hline Age 50-64 years & 30.03 & 30.32 & 0.01 \\
\hline Age 65-74 years & 13.10 & 12.28 & 0.03 \\
\hline Age $75-79$ years & 4.59 & 3.77 & 0.04 \\
\hline Age $\geq 80$ years & 5.95 & 5.42 & 0.02 \\
\hline Female sex & 56.19 & 55.66 & 0.01 \\
\hline Pulmonary embolism ( \pm deep vein thrombosis) & 18.09 & 17.89 & 0.01 \\
\hline \multicolumn{4}{|l|}{ Comorbidities } \\
\hline Chronic obstructive pulmonary disease & 8.37 & 7.98 & 0.01 \\
\hline Asthma & 13.25 & 13.10 & 0.00 \\
\hline Heart Failure & 4.84 & 4.93 & 0.00 \\
\hline Hypertension & 52.80 & 51.45 & 0.27 \\
\hline Ischemic stroke or transient ischemic attack & 2.95 & 2.85 & 0.01 \\
\hline Diabetes & 21.13 & 21.03 & 0.00 \\
\hline Dementia & 2.18 & 2.03 & 0.01 \\
\hline Coronary artery disease & 0.68 & 0.87 & 0.02 \\
\hline Carotid stenosis & 0.63 & 0.53 & 0.01 \\
\hline Peripheral vascular disease & 5.51 & 5.42 & 0.00 \\
\hline Myocardial infarction & 5.08 & 4.59 & 0.02 \\
\hline Percutaneous coronary intervention & 3.34 & 3.13 & 0.01 \\
\hline Coronary artery bypass grafting & 1.93 & 2.18 & 0.02 \\
\hline Gastrointestinal bleed & 0.24 & 0.29 & 0.01 \\
\hline Intracranial hemorrhage & 0.00 & 0.00 & NA \\
\hline Acute kidney injury & 10.06 & 9.38 & 0.02 \\
\hline Other kidney injury & 0.24 & 0.24 & 0.00 \\
\hline Inflammatory bowel disease & 0.77 & 0.82 & 0.01 \\
\hline eGFR $>90 \mathrm{~mL} /$ minute & 55.42 & 58.32 & 0.06 \\
\hline eGFR 60-89 mL/minute & 0.48 & 0.73 & 0.03 \\
\hline eGFR $30-59$ mL/minute & 31.09 & 28.97 & 0.05 \\
\hline eGFR 15-29 mL/minute & 10.88 & 10.06 & 0.03 \\
\hline eGFR $<15 \mathrm{~mL} /$ minute & 1.21 & 1.11 & 0.01 \\
\hline eGFR unknown & 0.77 & 0.68 & 0.01 \\
\hline Liver disease & 1.50 & 1.93 & 0.03 \\
\hline Coagulopathy & 3.00 & 3.00 & 0.00 \\
\hline Gastroesophageal reflux disease & 18.76 & 18.96 & 0.00 \\
\hline Anemia & 24.13 & 23.55 & 0.01 \\
\hline Sleep apnea & 10.20 & 10.64 & 0.01 \\
\hline Smoking & 28.77 & 28.19 & 0.01 \\
\hline Hemorrhoids & 2.22 & 2.37 & 0.01 \\
\hline Alcohol abuse & 0.34 & 0.34 & 0.00 \\
\hline Anxiety & 12.28 & 14.02 & 0.05 \\
\hline
\end{tabular}


Table 3 Characteristics of the 1:1 Propensity Score Matched (Sensitivity Analysis) Rivaroxaban and Warfarin Cohorts (Continued)

\begin{tabular}{|c|c|c|c|}
\hline & $\begin{array}{l}\text { Rivaroxaban } \\
N=2068 \\
\%\end{array}$ & $\begin{array}{l}\text { Warfarin } \\
N=2068 \\
\%\end{array}$ & $\begin{array}{l}\text { Absolute Standardized } \\
\text { Difference }\end{array}$ \\
\hline Depression & 1.69 & 1.60 & 0.01 \\
\hline Psychosis & 1.50 & 1.16 & 0.03 \\
\hline $\mathrm{BMI}<18.5 \mathrm{~kg} / \mathrm{m}^{2}$ & 1.60 & 1.74 & 0.01 \\
\hline BMI $18.5-24.9 \mathrm{~kg} / \mathrm{m}^{2}$ & 15.43 & 14.46 & 0.03 \\
\hline BMI $25.0-29.9 \mathrm{~kg} / \mathrm{m}^{2}$ & 24.71 & 25.29 & 0.01 \\
\hline BMI $30.0-34.9$ kg/m² & 23.26 & 23.79 & 0.01 \\
\hline BMI $35.0-39.9$ kg/m² & 14.70 & 15.47 & 0.02 \\
\hline $\mathrm{BMI} \geq 40 \mathrm{~kg} / \mathrm{m}^{2}$ & 19.15 & 17.65 & 0.04 \\
\hline BMI unknown & 1.16 & 1.60 & 0.03 \\
\hline Rheumatoid arthritis & 5.80 & 6.53 & 0.03 \\
\hline Osteoarthritis & 18.86 & 18.23 & 0.02 \\
\hline Headache & 10.15 & 10.59 & 0.01 \\
\hline Diverticulitis & 3.72 & 3.77 & 0.00 \\
\hline H. pylori treatment & 0.39 & 0.34 & 0.01 \\
\hline Hypothyroidism & 0.87 & 0.87 & 0.00 \\
\hline Solid tumor & 9.72 & 8.90 & 0.03 \\
\hline Metastatic cancer & 3.72 & 3.29 & 0.02 \\
\hline Major surgery & 10.11 & 10.01 & 0.00 \\
\hline Varicose veins & 1.26 & 1.35 & 0.01 \\
\hline \multicolumn{4}{|l|}{ Comedications } \\
\hline Aspirin & 22.10 & 21.47 & 0.02 \\
\hline P2Y12 platelet inhibitor & 2.90 & 2.80 & 0.01 \\
\hline Nonsteroidal anti-inflammatory drug & 31.33 & 33.95 & 0.05 \\
\hline Celecoxib & 1.02 & 1.35 & 0.03 \\
\hline Angiotensin-converting enzyme inhibitor or receptor blocker & 30.66 & 29.21 & 0.03 \\
\hline Beta-blocker & 22.44 & 21.52 & 0.02 \\
\hline Diltiazem & 1.55 & 1.69 & 0.01 \\
\hline Verapamil & 0.87 & 0.87 & 0.00 \\
\hline Dihydropyridine calcium channel blocker & 20.31 & 19.44 & 0.02 \\
\hline Loop diuretic & 10.69 & 9.91 & 0.03 \\
\hline Thiazide & 21.08 & 20.45 & 0.02 \\
\hline Digoxin & 0.44 & 0.39 & 0.01 \\
\hline Statin & 23.02 & 21.66 & 0.03 \\
\hline Other cholesterol medication & 2.13 & 1.98 & 0.01 \\
\hline Metformin & 11.41 & 11.17 & 0.01 \\
\hline Sulfonylurea or glinides & 4.64 & 4.30 & 0.02 \\
\hline Thiazolidinediones & 0.34 & 0.53 & 0.03 \\
\hline Dipeptidyl peptidase 4 inhibitors & 1.35 & 1.64 & 0.02 \\
\hline Glucagon-like peptide-1 agonist & 0.29 & 0.44 & 0.02 \\
\hline Insulin & 7.54 & 7.06 & 0.02 \\
\hline Selective serotonin reuptake or serotonin-norepinephrine reuptake inhibitor & 10.88 & 10.74 & 0.00 \\
\hline Other antidepressants & 8.95 & 8.80 & 0.01 \\
\hline Proton pump inhibitors & 21.23 & 21.03 & 0.00 \\
\hline
\end{tabular}


Table 3 Characteristics of the 1:1 Propensity Score Matched (Sensitivity Analysis) Rivaroxaban and Warfarin Cohorts (Continued)

\begin{tabular}{llll}
\hline & $\begin{array}{l}\text { Rivaroxaban } \\
N=2068 \\
\%\end{array}$ & $\begin{array}{l}\text { Warfarin } \\
\text { \% }=2068 \\
\%\end{array}$ & $\begin{array}{l}\text { Absolute Standardized } \\
\text { Difference }\end{array}$ \\
\hline Histamin-2 receptor antagonist & 9.14 & 8.46 & 0.02 \\
Systemic corticosteroids & 18.13 & 18.09 & 0.00 \\
Alpha-glucosidase inhibitor & 0.00 & 0.00 & 0.00 \\
Hypnotic medication & 3.68 & 4.01 & 0.02 \\
Sodium-glucose cotransporter-2 inhibitor & 0.15 & 0.19 & 0.01 \\
\hline
\end{tabular}

our intention-to-treat analysis should be interpreted with caution as it is unclear how accurately EHRs maintain up-to-date patient medication profiles. Moreover, an EHR entry to initiate an OAC does neither guarantee a patient took the medication nor (as in claim data sets) assures patients even picked up their prescription at the pharmacy. Finally, absent randomization in a study, residual confounding cannot be fully excluded due to the possibility of confounding from unobserved or unmeasured covariates [11].

\section{Conclusions}

In conclusion, our EHR-based study suggests rivaroxaban is no worse than warfarin at preventing recurrent VTE with no increased risk of major bleeds in African Americans. Our findings are similar to those found in the EINSTEIN clinical trials and consistent with the results from the XALIA study. Additional real-world analyses with an increased sample size to evaluate the effectiveness and safety of rivaroxaban for treatment and prevention of VTE in African Americans are warranted.

Table 4 Results of Sensitivity Analyses

\begin{tabular}{|c|c|c|}
\hline & 1:1 Propensity Score Matching & $\begin{array}{l}\text { On- } \\
\text { Treatment } \\
\text { Approach }\end{array}$ \\
\hline \multicolumn{3}{|l|}{ 3-Month } \\
\hline $\begin{array}{l}\text { Composite of recurrent venous } \\
\text { thromboembolism or major bleeding }\end{array}$ & $1.10(0.82-1.46)$ & $1.10(0.83-1.45)$ \\
\hline Recurrent venous thromboembolism & $1.08(0.78-1.50)$ & $1.11(0.81-1.52)$ \\
\hline Major bleeding & $1.28(0.73-2.25)$ & $1.17(0.69-1.98)$ \\
\hline Intracranial hemorrhage & $1.04(0.15-7.37)$ & $0.65(0.12-3.47)$ \\
\hline Gastrointestinal bleeding & $1.11(0.56-2.19)$ & $1.08(0.55-2.13)$ \\
\hline Genitourinary bleeding & $1.39(0.31-6.21)$ & $1.05(0.29-3.75)$ \\
\hline \multicolumn{3}{|l|}{ 6-Month } \\
\hline $\begin{array}{l}\text { Composite of recurrent venous } \\
\text { thromboembolism or major bleeding }\end{array}$ & $1.00(0.767-1.31)$ & $1.05(0.81-1.37)$ \\
\hline Recurrent venous thromboembolism & $1.04(0.76-1.41)$ & $1.12(0.83-1.53)$ \\
\hline Major bleeding & $1.02(0.62-1.69)$ & $1.01(0.62-1.66)$ \\
\hline Intracranial hemorrhage & $0.70(0.12-4.20)$ & $0.65(0.12-3.47)$ \\
\hline Gastrointestinal bleeding & $0.80(0.43-1.47)$ & $0.94(0.50-1.78)$ \\
\hline Genitourinary bleeding & $1.39(0.31-6.21)$ & $0.85(0.25-2.84)$ \\
\hline \multicolumn{3}{|l|}{ 12-Month } \\
\hline $\begin{array}{l}\text { Composite of recurrent venous } \\
\text { thromboembolism or major bleeding }\end{array}$ & $0.98(0.76-1.25)$ & $1.04(0.80-1.34)$ \\
\hline Recurrent venous thromboembolism & $1.00(0.75-1.34)$ & $1.10(0.82-1.47)$ \\
\hline Major bleeding & $0.99(0.64-1.5)$ & $0.98(0.61-1.57)$ \\
\hline Intracranial hemorrhage & $0.94(0.29-3.10)$ & $0.82(0.21-3.30)$ \\
\hline Gastrointestinal bleeding & $0.84(0.47-1.48)$ & $0.88(0.47-1.65)$ \\
\hline Genitourinary bleeding & $1.34(0.36-5.00)$ & $1.03(0.33-3.24)$ \\
\hline
\end{tabular}




\section{Abbreviations}

Cl: Confidence intervals; DVT: Deep vein thrombosis; EHR: Electronic health record; HR: Hazard ratio; ICH: Intracranial hemorrhage; IPTW: Inverse probability-of-treatment weighting; Gl: Gastrointestinal; GU: Genitourinary; NOACs: Non-vitamin K oral anticoagulants; OAC: Oral anticoagulant; PE: Pulmonary embolism; RCTs: Randomized controlled trials; RECORD: Reporting of studies conducting using observational routinelycollected health data; US: United States; VTE: Venous thromboembolism

\section{Acknowledgements}

Data used in this study were obtained from the Optum Inc. under a license to Janssen Scientific Affairs LLC (and provided to Dr. Coleman under a thirdparty agreement) and are not publicly available.

\section{Authors' contributions}

C. I. Coleman, V. Ashton, and M. Palladino conceptualized and designed the study. O. S. Costa, C. I. Coleman and T. J. Bunz analyzed the data. O. S. Costa, C. I. Coleman, S. Thompson, V. Ashton, M. Palladino, T. J. Bunz interpreted the data. The manuscript was written primarily by O. S. Costa and C. I. Coleman; all remaining authors aided and/or contributed to revisions. All authors substantially contributed to this project, read and approved the manuscript and assume responsibility for the contents of the manuscript.

\section{Funding}

This study was funded by Janssen Scientific Affairs LLC.

\section{Availability of data and materials}

Data used in this study were obtained from Optum under a license to Janssen Scientific Affairs LLC (and provided to Dr. Coleman under a third-party agreement) and are not publicly available.

\section{Ethics approval and consent to participate}

The use of the Optum EHR database was reviewed by the New England Institutional Review Board (IRB) and was determined to not constitute research involving human subjects according to 45 Code of Federal Regulations 46.102(f) and therefore deemed exempt from board oversight.

\section{Consent for publication}

Not applicable.

\section{Competing interests}

C. I. Coleman has received grant funding and consultancy fees from Janssen Scientific Affairs LLC, Titusville, NJ and Bayer AG, Berlin, Germany and speaker fees from Medscape Inc.

V. Ashton is an employee of Janssen Scientific Affairs LLC, Titusville, NJ. M. Palladino is an employee of Janssen Pharmaceuticals Inc., Titusville, NJ. S. Thompson serves on the Xarelto Speaker's Bureau for Janssen Pharmaceuticals, Titusville, NJ and advisory boards for Salix Pharmaceuticals, Bridgewater, NJ.

O. S. Costa and T. J. Bunz have no declarations-of-interest.

\section{Author details}

${ }^{1}$ Department of Pharmacy Practice, University of Connecticut School of Pharmacy, 69 North Eagleville Road, Unit 3092, Storrs, CT 06269, USA.

${ }^{2}$ Evidence-Based Practice Center, Hartford Hospital, Hartford, CT, USA.

${ }^{3}$ TeamHealth LifePoint Group, Southaven, MS, USA. ${ }^{4}$ Real World Value and Evidence, Janssen Scientific Affairs LLC, Titusville, NJ, USA. ${ }^{5}$ Medical Affairs, Janssen Pharmaceuticals Inc., Titusville, NJ, USA. ${ }^{6}$ Department of Pharmacoepidemiology, New England Health Analytics LLC, Granby, CT, USA.

Received: 19 December 2019 Accepted: 18 March 2020 Published online: 08 April 2020

\section{References}

1. White RH, Zhou H, Romano PS. Incidence of idiopathic deep venous thrombosis and secondary thromboembolism among ethnic groups in California. Ann Intern Med. 1998;128:737-40.

2. White RH, Zhou H, Murin S, Harvey D. Effect of ethnicity and gender on the incidence of venous thromboembolism in a diverse population in California in 1996. Thromb Haemost. 2005;93:298-305.
3. Schneider $\mathrm{D}$, Lilienfeld $\mathrm{DE}, \mathrm{Im} \mathrm{W}$. The epidemiology of pulmonary embolism: racial contrasts in incidence and in-hospital case fatality. J Natl Med Assoc. 2006;98:1967-72.

4. Dowling NF, Austin H, Dilley A, Whitsett C, Evatt BL, Hooper WC. The epidemiology of venous thromboembolism in Caucasians and AfricanAmericans: the GATE study. J Thromb Haemost. 2003;1:80-7.

5. Jackson LR 2nd, Peterson ED, Okeagu E, Thomas K. Review of race/ethnicity in non vitamin $\mathrm{K}$ antagonist oral anticoagulants clinical trials. J Thromb Thrombolysis. 2015;39:222-7.

6. Di Nisio M, Ageno W, Rutjes AW, Pap AF, Büller HR. Risk of major bleeding in patients with venous thromboembolism treated with rivaroxaban or with heparin and vitamin K antagonists. Thromb Haemost. 2016;115:424-32.

7. Agnelli G, Buller HR, Cohen A, et al. Oral apixaban for the treatment of acute venous thromboembolism. N Engl J Med. 2013;369:799-808.

8. Buller HR, Decousus H, Grosso MA, et al. Edoxaban versus warfarin for the treatment of symptomatic venous thromboembolism. N Engl J Med. 2014; 369:1406-15.

9. Prins $\mathrm{MH}$, Lensing $\mathrm{AW}$, Bauersachs $\mathrm{R}$, et al. Oral rivaroxaban versus standard therapy for the treatment of symptomatic venous thromboembolism: a pooled analysis of the EINSTEIN-DVT and PE randomized studies. Thromb J. 2013;11:21.

10. Schulman S, Kearson C, Kakkar AK, et al. Dabigatran versus warfarin in the treatment of acute venous thromboembolism. N Engl J Med. 2009;361: 2342-52.

11. Optum. Optum EHR Offering. Optum Inc, 2018. Available at: https://www. optum.com/campaign/ls/data-new-era-of-visibility/download.html (Last accessed on July 28, 2019).

12. White RH, Garcia M, Sadeghi B, et al. Evaluation of the predictive value of ICD-9-CM coded administrative data for venous thromboembolism in the United States. Thromb Res. 2010;126:61-7.

13. Cunningham A, Stein CM, Chung CP, Daugherty JR, Smalley WE, Ray WA. An automated database case definition for serious bleeding related to oral anticoagulant use. Pharmacoepidemiol Drug Saf. 2011;20:560-6.

14. Austin PC. An introduction to propensity score methods for reducing the effects of confounding in observational studies. Multivariate Behav Res. 2011;46:399-424.

15. Benchimol El, Smeeth L, Guttmann A, Harron K, Moher D, Petersen I, Sørensen HT, von Elm E, Langan SM. RECORD Working Committee. The REporting of studies Conducted using Observational Routinely-collected health Data (RECORD) statement. PLoS Med. 2015;12:e1001885.

16. Ageno W, Mantovani LG, Haas S. Safety and effectiveness of oral rivaroxaban versus standard anticoagulation for the treatment of symptomatic deep-vein thrombosis (XALIA): an international prospective, non-interventional study. Lancet Haematol. 2016;3:e12-21.

17. Gandhi SK, Salmon W, Kong SX, Zhao SZ. Administrative databases and outcomes assessment: an overview of issues and potential utility. J Manag Care Spec Pharm. 1999;5:215-22.

18. Coleman Cl, Turpie AG, Bunz TJ, Beyer-Westendorf J. Effectiveness and safety of rivaroxaban versus warfarin in patients with provoked venous thromboembolism. J Thromb Thrombolysis. 2018:46:339-45.

19. Erkens PM, ten Cate H, Büller HR, Prins MH. Benchmark for time in therapeutic range in venous thromboembolism: a systematic review and meta-analysis. PLoS One. 2012;7:e42269. https://doi.org/10.1371/journal. pone.0042269.

20. Limone BL, Hernandez AV, Michalak D, Bookhart BK, Coleman Cl. Timing of recurrent venous thromboembolism early after the index event: a metaanalysis of randomized controlled trials. Thromb Res. 2013;132:420-6.

21. Yong C, Xu X, Than C, Ullal A, Schmitt S, Azarbal F, Heidenreich P, Turakhia $M$. Racial disparities in warfarin time in INR therapeutic range in patients with atrial fibrillation: findings from the TREAT-AF study. Circulation. 2013; 128:A14134.

\section{Publisher's Note}

Springer Nature remains neutral with regard to jurisdictional claims in published maps and institutional affiliations. 